\title{
Commentary
}

\section{Microchimerism, macrochimerism, and tolerance}

This work was supported by the National Institutes of Health grants DK 29961. R01 Al038899. and R01 DK 54232.

The meta-analysis of Sahota et al. (1) suggests that donor leukocyte microchimerism in organ recipients is a 'real finding' that varies with the organ transplanted and the time of post-operative sampling. and is detectable with the appropriate technology from center to center. The meta-analysis also reflects the lack of consensus about the signifcance of microchimerism. This uncertainty has been perpetuated in part by incorrectly attributing to us the opinion that microchimerism implies freedom from risk of rejection and or that its presence or level can be used to guide drug weaning. Sahota et al. (1) have avoided these errors. Nevertheless. inaccurate citations to this effect (2) have been used secondarily to support the contention that the mechanisms of organ engraftment are different than those of bone marrow-induced tolerance (35).

The clinical field of organ transplantation is based on the fact that a host response against the graft. which may or may not be strong enough to cause clinical and or histopathologic findings of rejection. is readily reversible and often is succeeded by a decline in the antigraft reactivity that is reflected by a reduced need for immunosuppression (6).

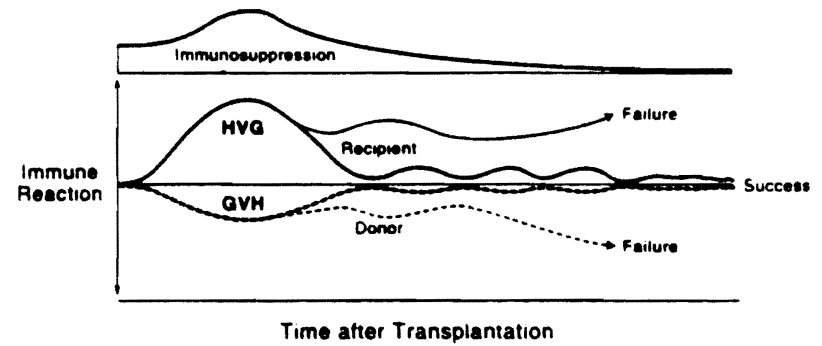

Fig. 1. Contemporaneous host-versus-graft (HVG) and graftversus-hosı (GVH) reactions after transplantation. Failure is defined as the inability to control one of the reactions, or sometimes both. Acute reciprocal clonal exhaustion after successful transplantation is maintained subsequently by chimerism-dependent low-grade stimulation of both leukocyte populations that may wax and wane. (By permission of New England Journal of Medicine: 339:1905-1913. 1998.)
The alteration in the host-graft relationship implied by these observations remained unknown until it was discovered in patients with long-surviving organ allografts that bone marrow-derived passenger leukocytes migrated from the allografts to ubiquitous host locations and persisted for as long as three subsequent decades (7.8).

From these findings. we deduced that the chimerism-associated mechanisms of organ engraftment involved a double-immune reaction (Fig. 1) and were the same in principle as those leading to tolerance following bone marrow transplantation to cytoablated recipients (7-9). Furthermore. it was emphasized that chimerism. whether at a 'micro" or "macro' level was only a necessary condition for. but was not synonymous with. either allograft acceptance or tolerance. This concept has been strengthened by a series of experimental stud. ies $(10-13)$ and has readily accommodated and explained observations made by its critics $(14,15)$.

The host-versus-graft (HVG) reaction after either kind of transplantation is analogous to the adaptive immune response to intracellular noncytopathic microorganisms (16-19) (Fig. 2). However, the consequences are more complex than those of a host versus pathogen response because of the countervailing graft-versus-host $(\mathrm{GVH})$ reaction mounted by mobile immunocompetent passenger leukocytes of the allograft (Fig. 1). We have proposed that the HVG and GVH immune reactions are regulated after both organ and conventional bone marrow transplantation by the migration and localization of the respective immunogenic leukocytes (19).

To induce an effective response. the antigen must be delivered to organized lymphoid collections that are epitomized by. but not limited to. the lymphoid organs (18. 19). In this milieu. factors are present in abundance that are necessary for efficient immune activation (e.g. cytokines. other molecules, cell to cell proximity) (18). After transplantation. the donor 'passenger leukocytes' leave 


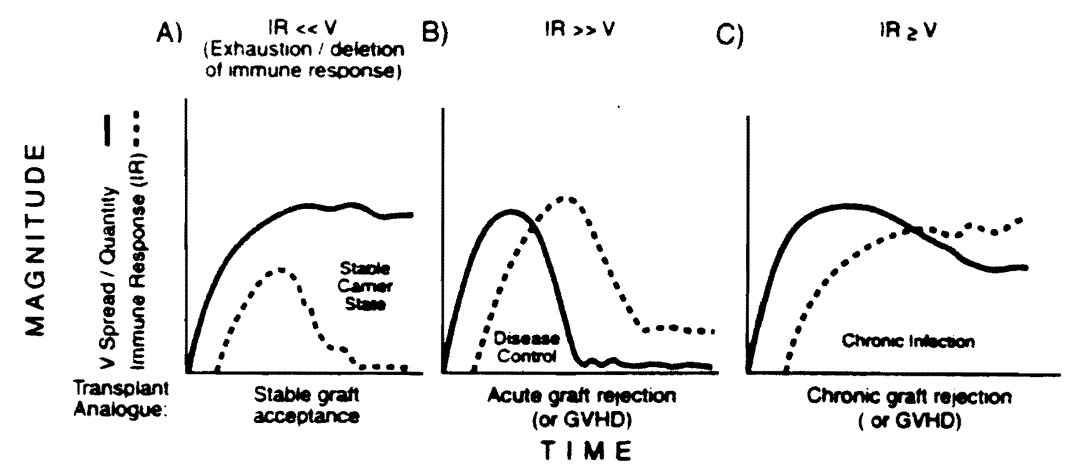

Fig. 2. Potential outcomes after infection with non-cytopathic microorganisms and analogies (expressed as rejection or graft-versushost disease) to organ transplantation. The horizontal axis denotes time. The vertical axis shows the magnitude of the viral load (v. solid line). and the host immune response (IR. dashed line). GVHD denotes graft-versus-host disease.

the allograft. and migrate preferentially to the recipient lymphoid organs (20-22). If an antigen primarily bypasses or secondarily avoids organized lymphoid collections. the immune system may remain or become 'indifferent' to its presence (1719).

In addition to governing the initiation of the double-immune response. antigen migration and localization regulate the termination of the dual response (19). It is well known that the GVH reaction usually is the stronger one in the cytoablated bone marrow recipient. whereas the dominant response in the organ recipient is usually HVG. If either immunocyte population completely rejects the other. the IL2 production of the surviving leukocyte cohort ceases and its antigen-specific clonal expansion is terminated by apoptosis. This 'antigen withdrawal' apoptosis requires new protein synthesis. is strongly inhibited by $\mathrm{Bcl}-2$ and related anti-apoptotic molecules. and is thought to involve mitochondrial apoptosis mechanisms rather than the death cytokines such as Fas ligand (FasL) and tumor necrosis factor (TNF) [summarized in (23)].

Alternatively. persistence of the dual sources of antigen may drive the double-immune reaction to mutual clonal exhaustion-deletion (7. 8. 19), involving different molecular pathways of apoptosis including FasL and TNF (23). The consequence of this kind of apoptosis may be reciprocal antigenspecific clonal exhaustion-deletion (24-26). Although the exhaustion-deletion is probably never absolute. it is thought to be maintained after successful transplantation in a dynamic but potentially stable state by the periodic 'leakage' of leukocytes from non-lymphoid to lymphoid areas (18. 19). as has been described in an experimental model of diabetes (27). In tissue and organ recipients. the mobile antigen (i.e. the donor leukocyte microchimerism) rather than the 'fixed' parenchy- mal cells of a graft is essential for the maintenance of tolerance (13). If the MHC-restricted HVG reaction to a transplanted organ is not terminated either by the induction of donor-specific tolerance (Fig. 2A). or by rejection of the allograft (Fig. 2B). the response becomes unrelenting and results in chronic rejection (Fig. 2C).

Discovery of the linkage between hematolymphopoietic chimerism and organ transplantation prompted the development of a paradigm that has challenged multiple dogmas of transplantation and general immunology, while explaining previously enigmatic observations in clinical transplantation $(7-9.15,19)$. At a basic level. it has been possible for the first time to define precisely the meaning of 'transplantation tolerance' and the mechanisms by which it is achieved (i.e. clonal exhaustion-deletion and immune indifference) (19). In a therapeutic context. it is obvious why it has been so difficult in HLA mismatched human organ recipients to achieve the closely related objectives of drug-free tolerance and freedom from chronic rejection.

In order to avoid losing allografts to acute rejection. intense immunosuppression is administered during the first few post-operative weeks of the acute donor-specific clonal expansion set in motion by the migration into the host of immunostimulatory donor passenger leukocytes. Because donorspecific clonal exhaustion-deletion is dependent on the acute clonal activation. the early post-transplantation period provides the prime window of opportunity for tolerance induction $(7,8)$. While saving the graft from rejection. the penalty for eroding the seminal mechanism of tolerogenesis with heavy immunosuppression may be the inability to ever stop drug treatment. Under these circumstances, chronic rejection supervenes if the maintenance doses are reduced below the critical threshold necessary to compensate for the incompleteness of the original tolerance induction. 
When it was recognized that the donor passenger leukocytes of bone marrow origin were responsible for organ-induced tolerance. efforts began in earnest to augment the natural process by the infusion of adjunct donor bone marrow cells (28) as had long been advocated empirically by Monaco et al. (29). However, because this has required the same fundamentally anti-tolerogenic immunosuppression as for conventional organ transplantation. the results have been disappointing as is apparent from the meta-analysis of Sahota et al (1). The low-level chimerism normally found in organ recipients has been increased manifold (28. 30. 31). and has been reported in some studies to correlate with a higher incidence of donor-specific non-reactivity. However. discontinuance of immunosuppression has not been achieved.

Finding the optimal zone between under-treatment (with consequent destructive immunity) and over-treatment (with excessive erosion of tolerogenesis) is the channel between Scylla and Charybdis that is being sought by workers in transplantation. The task has been made more daunting by the fact that the appropriate conditions. unlike those in inbred animal tolerance models. are never exactly the same in any two human recipients. It is possible that clonal exhaustion-deletion can be accomplished more efficiently with new agents such as the monoclonal antibodies that block co-stimulation and therefore prevent clonal expansion at a very early stage $(32-34)$. However. the basic requirement for. and mechanisms of. acquired tolerance will remain the same.

Thomas E Star=l Noriko Murase

\section{References}

1. Sahota a. Gao S. hayes J. Jindal RM. Microchimerism and rejection: a meta-analysis. Clin Transplant 2000: 14: 345 .

2. Starzl te. Mlirase N. Demetris aJ. Qian S Microchimerism: inaccurate literature citations. Transplantation 1996: 62: 703.

3. WOOD K. SACHS DH. Chimerism and transplantation tolerance: cause and effect. Immunol Today 1996: 17: 584.

4. WeKerle T. SYKes M. Mixed chimerism as an approach for the induction of transplantation tolerance Transplantation 1999: 68: 459

5. Bl'shell A. Pearson tC. Morris PJ. WoOd KJ Donor-recipient microchimerism and tolerance induction Transplantation 1996: 61: 170.

6. Starzl te. Marchioro Tl. Waddell W'R. The reversal of rejection in human renal homografts with subsequent development of homograft tolerance. Surg Gynecol Obstet 1963: 117: 385.
7. Starzl te. Demetris aj. Mlrase N. Ildstad S. RICORDI C. TRLCCO M. Cell migration. chimerism. and graft acceptance. Lancet 1992: 339: 1579

8. Starzl te. Demetris aj. Trlcco $M$ et al. Cell migration and chimerism after whole-organ transplantation: the basis of graft acceptance. Hepatology 1993: 17: 1127

9. Starzl TE. Demetris AJ. Transplantation milestones: viewed with one- and two-way paradigms of tolerance. J Am Med Assoc 1995: 273: 876.

10. Deimetris aj. Mlirase N. Fluisaki S. Fling Jj. Rao AS. STARZL TE. Hematolymphoid cell trafficking. microchimerism. and GVHD reactions after liver. bone marrow. and heart transplantation. Transplant Proc 1993 25: 3337

11. Qian S. Demetris aj. Mlrase N. Rao aS. Fleng JJ. STARZL TE. Murine liver allograft transplantation: tolerance and donor cell chimerism. Hepatology 1994: 19: 916

12. Mirase $i$ Starzl te. Tavabe $M$ et al. Variable chimerism. graft versus host disease. and tolerance after different kinds of cell and whole organ transplantation from Lewis to Brown- Norway rats. Transplantation 1995: 60: 158 .

13. Ehl S. Aichele P. Ranseier $\mathrm{H}$ et al. Antigen persistence and time of T-cell tolerization determine the efficacy of tolerization protocols for prevention of skin graft rejection. Nat Med 1998: 4: 1015

14. Starzl te. Rao as. Thomson aw. Mlrase N. DEMETRIS AJ. Donor-recipient microchimerism and tolerance induction (Letter to the Editor). Transplantation 1996: $61: 169$

15. Starzl te. Demetris aj. Mlrase N. Trlcco M. THOMSON AW. RAO AS. The lost chord: microchimerism. Immunol Toda! 1996: $1^{-1: 577}$

16. Doherty PC. ZiNkerNagel R.M. A biological role for the major histocompatibility antigens. Lancet 1975: $\mathrm{i}$ 1406.

17. ZINKERNAGEL RM. Immunology taught by viruses Science 1996: 271: 173.

18. Zinkernagel RM. Ehl S. Aichele P. Oehen S. KLNDIG T. HENGARTNER $H$. Antigen localization regulates immune responses in a dose- and time-dependent fashion: a geographical vieu of immune reactivity. Immunol Rev 1997: 156: 199.

19. StARZL TE. ZiNkER NaGel RM. Antigen localization and migration in immunity and tolerance. New Engl J Med 1998: 339: 1905.

20. Nemlander A. Soots A. Willebrand EV. Husberg B. HAY'RY P. Redistribution of renal allograft responding leukocytes during rejection. II. Kinetics and specificity. J Exp Med 1982: 156: 1087 .

21. LARSEN CP. MORRIS PJ. ALSTIX JM. Migration of dendritic leukocytes from cardiac allografts into host spleens: a novel route for initiation of rejection. J Exp Med 1990: 17.1: 307.

22. Demetris AJ. QiaN S. Si $\mathbf{N} \mathrm{H}$ et al. Early events in liver allograft rejection: delineation of sites simultaneous intragraft and recipient lymphoid tissue sensitization. Am J Pathol 1991: 138: 609

23. Levardo M. Chan FKM. Horning F et al. Mature T lymphocyte apoptosis - immune regulation in a dynamic and unpredictable antigenic environment. Annu Rev Immunol 1999: 17: 221.

24. QIAN S. LL L. FL: F et al. Apoptosis within spontaneously accepted mouse liver allografts: evidence for deletion of crtotoxic $T$ cells and implications for tolerance induction. J Immunol 1997: 158: 4654. 
25. LU L, L1 W, ZHONG $\mathrm{C}$ et al. Increased apoptosis of immunoreactive host cells and augmented donor leukocyte chimerism. not sustained inhibition of B7 molecule expression are associated with prolonged cardiac allograft survival: in mice preconditioned with immature donor dendritic cells plus anti-CD40L mAb. Transplantation 1999: 68: 747.

26. Wekerle T. Sayegh MH. Hill $J$ et al. Extrathymic $T$ cell deletion and allogeneic stem cell engraftment induced with costimulatory blockade is followed by central $T$ cell tolerance. J Exp Med 1998: 187: 2037.

27. OHASHI PS. OEHEN S. BLERKI K et al. Ablation of tolerance and induction of diabetes by viruses infection in viral antigen transgenic mice. Cell 1991: 65: 305.

28. Fontes P. R.to A. Demetris AJ et al. Augmentation with bone marrow of donor leukocyte migration for kidney, liver. heart. and pancreas islet transplantation. Lancet 1994: 344: 151.

29. Monaco AP. ClaRK AW. Brown RW. Active enhancement of a human cadaver renal allograft with ALS and donor bone marrow: case report of an initial attempt. Surgery 1976: 79: 384.

30. Salgar S. Shapiro R. Dodson F et al. Infusion of donor leukocytes to induce tolerance in organ allograft recipients. J Leukoc Biol 1999: 66: $\$ 10$.
31. Garcia Morales R. Esquenazi V. Zlicker $\mathrm{K}$ et al. Assessment of the effects of cadaver donor bone marrow on kidney allograft recipient blood cell chimerism by a novel technique combining PCR and flow cytometry (PCR-FLOW). Transplantation 1996: 62: 1149.

32. LARSEN CP. ElwoOd ET. Alexander DZ et al. Long-term acceptance of skin and cardiac allografts after blocking CD40 and CD28 pathways. Nature 1996: 381: 434 .

33. Kirk AD. HaRlan DM. ARMSTRONG NN. Davis TA DONG Y. Gray GS. HONG X. Thomas D. FechNer JH JR.. K.NECHTLE SJ. CTLA4-Ig and anti-CD40 ligand prevent renal allograft rejection in primates. Proc Natl Acad Sci USA 1997: 8789

34. Wells AD. Li XC. Li Y et al. Requirement for T-cell apoptosis in the induction of peripheral transplantation tolerance. Nat Med 1999: 5: 1303.

Correspondence:

Thomas Starzl

Department of Surgery

University of Pittsburgh

PA 15213

LSA 